fore 30 June and details are obtainable from I.N.S.E.R.M., c/o Mlle. Ladeveze, 3 rue Léon Bonnat, Paris XVI, France.

\section{Anaesthetics Prize}

The Royal Society of Medicine, Section of Anaesthetics, invites applications for a prize of $£ 100$ provided by Messrs. May and Baker Ltd. for a paper written by a senior registrar or registrar holding an appointment in anaesthesia in a department or hospital, or a doctor of similar status in the armed Forces of the Commonwealth or of the Republics of Ireland or South Africa. Further details may be obtained from the sections officer, Royal Society of Medicine, 1 Wimpole Street, London WIM 8AE.

\section{Beit Memorial Fellowships}

The trustees of the Beit Memorial Fellowships for medical research have announced the following elections:

funior Fellowship: R. C. Atkins (M.R.C. Cellular Immunology Unit, Sir William Dunn School of Pathology, Oxford University); $M$. Burrows (department of zoology, Oxford University); E. B. Gingold (department of biochemistry, School of Biological Sciences, Leicester University); S. B. Hladky (physiological laboratory, Cambridge University); M. C. Gregory (department of the regius professor of medicine, Radcliffe Infirmary, Oxford); C. W. Lloyd (Strangeways Research Laboratory, Cambridge); A. R. Michell (department of medicine, Royal Veterinary College, London University); J. B. Furness (department of physiology, Birmingham University Medical School); A. L. Lehmann (department of biochemistry, Sussex University).

\section{People in the News}

Dr. Douglas Gordon, who has been doing ultrasonic diagnostic work at Moorfields Eye Hospital, London, for some years, has been elected an honorary fellow of the American Institute of Ultrasound in Medicine.

\section{COMING EVENTS}

Royal College of Physicians of London.-Clinical physiology session, "Hirsutism," 22 June, 2.30 p.m. See advertisement on page xvi.

Cambridge Graduates Medical Club.-Annual general meeting and annual dinner, 9 July, 7 p.m., Downing College. Tickets (£3.75) and details from Dr. H. H. E. Batten, 67 Bridge Street, Cambridge.

Family Planning Association.-National Conference, "New Frontiers of Birth Control," 2022 July, Royal Festival Hall. Details and registration forms (closing date 18 June) are obtainable from the conference secretariat, F.P.A., 27-35 Mortimer Street, London W1A 4QW. (Tel. 01-636 7866.)

\section{SOCIETIES AND LECTURES}

For attending lectures marked a fee is charged or a ticket is required. Applications
made first to the institution concerned.

Monday, 14 June

INSTTTUTE OF DeRMatology. -4.30 p.m., Dr. Y. M. Clayton: Therapy of Fungal Infections.
INSTITUTB OF LARYNGOLOGY AND OTOLOGY.-5.30 p.m., combined consultation clinical meeting.

Tuesday, 15 June

INSTITUTE OF Dermatology. -4.30 p.m., Professor
Westminster Medical SCHOol.-5.15 p.m., Mr. J. Colin, Mr. C. Wastell: Effect of
Alcohol on Ability to Drive a Car.

\section{Wednesday, 16 June}

Royal Postgraduate Medical SCHOOl.-2 p.m. Dr. B. Rowe: Enteropathogenic $E$. coli

p.m. Dr. A C Stevenson: Chromosommary, Arising in Course of Treatment of Rheumatic

Royal College of Obstetricians and GynaecoloGISTS. - 5.30 p.m., postgraduate museum demonstration by Dr. Christine M. Davidson: Cytolog

\section{Thursday, 17 June}

Royal College of Surgeons of England. - 5 p.m. Arris and Gale lecture, Mr. J. M. Beaugie:

ST. MARY's Hospital MEDICAL SCHOOL. 5.15 p.m., Mr. Stirrat: The Fetus as a Homograft.

Royal Society OF Tropical MEDICINE AND HyGIENE. -8 p.m., Professor D. S. Bertram: Bugs,

Friday, 18 June

Royal Postgraduate Medical School.--11 a.m. Mr. J. E. A. Wickham: Regional Hypothermia in Conservative Renal Surgery.

CWCASTLE UPON TYNE REgIONAL NeUROLOGICA p.m., Professor S. Shuster; Parkinsonism, Pimples and the Pituitary.

\section{UNIVERSITIES AND COLLEGES}

\section{LONDON}

M.D.-G. A. Coles, E. G. J. Olsen, J. E. Stevens THIRD M.B., B.S. ${ }^{1}{ }^{3}$ Rosemary J. Basson, ${ }^{15}$ A. J. Camm, medal), $14 \mathrm{~J}$. F. Dracass, ${ }^{4} \mathrm{M}$. S. S. Fletcher, ${ }^{15} \mathrm{D}$. M

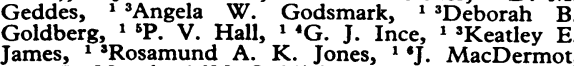
James, R. Mundy, 1 'M. S. Nielsen, ' 'Susan R. Rudge, H. W. Aitken, I. D. Almond, Janet E. F. A. Warne, H. W. Aitken, I. D. Almond, Janet E. F. Anderman,
Nellie T. Annan, D. J. Anton, P. Arnison-Newgass, Sally M. Arnold, Sarah R. Atkinson, G. R. Avery, J. R. Bailey, M. J. Bailey, H. R. Baillie-Johnson,
C. C. Baker, Ann F. Barker, P. A. Barker, A. J. Barnes
Anne P. M. P. Barnes, R. K. Barnes, D. J. A. Bateman J. L. Bates, P. H. Bavister, A. K. Beavis, H. S. Beesley, R. G. A. Behrman, D. Bellamy, J. O. Beltran, Patricia M. A. H. C. Boddam-Whetham, A. R. J. Bosley, I. R Bowen, G. C. Brabbins, G. V. H. Bradby, F. J. Branicki, P. V. Buckingham, G. C. Budden, M. Burke, T. M Burnett, Angela S. Butler, C. W. Caldwell, Joan CallenRoss, P. S. Cary, P. C. G. Chan-Kam, D. W. Chester, C. P. Ciclitira, Janet A. Clements, M. E. J. Cole, S. R. D. P. J. Ciclitira, Janet A. Clements, M. E. J. Cole, S. R. D.
Coles, R. J. Collins, S. M. Collins, S. J. Connellan,
R. W. I. Cooke, R. C. D. S. Coombes, M. J. Cooper, R. W. I. Cooke, R. C. D. S. Coombes, M. J. Cooper
Irene D. Cormac, T. M. Cox, Hilary L. Coysh, Patrici A. Crawford, Barbara J. Crofts, N. I. Dale, Margaret J. Davidson, Gillian V. Davies, S. G. Dawson, J. P. Delamere, T. F. Dent, Susan E. Dinnick, Gillian Dodds, Gillean P. Duncan, D. B. Dunger, D. A. B. Dunlop, Topan Dutta, J. P. F. Edmonds, K. S. Ellam, C. J. Ellis, Fan-Lun, R. Fawkner-Corbett, Pamela C. Fenney,
R. S. Fink, I. R. Fletcher, Sandra A. Floyd, Patrici Fox-Male, Stephanie R. French, G. J. Froyd, R. H. S
Furness, R. M. Galbraith, G. R. Gallimore, Elizabeth A. S. Galvin, M. D. Gambling, M. H. Garland, A. D
Garner, D. J. Giraldi, M. G. Glaser, R. E. Glass Jenifer I. Glover, D. Goldmeier, I. C. Goodrick, P. R. Goodwin, J. W. W. Gothard, J. J. Graham, Greenberg, I. W. Grier, Mary Griffin, R. A. Gude, J. F. Hamlyn, S. R. Hammond, M. H. Hampton, B. G. Hands, J. G. G. Hanley, I. Hardy, J. D. Harries,
G. P. Harris, P. R. Harrison, J. E. Harvey, P. B. Harvey, T. W. Higenbottam, B. M. Higginson, Alison P. Hill Holgate, S. O. Holland, K. G. Hollebone, N. Holmes, Sheilagh M. Hope, S. D. Horsley, D. C. Horsman D. J. Houghton, N. B. Houghton, J. R. Hughes, M. D
Humby, K. R. Hyde, P. B. Iles, C. R. Jagger, B. G
James, S. W. Jamieson, W. J. Jarratt, Celia A. Jenkins, James, S. W. Jamieson, W. J. Jarratt, Celia A. Jenkins, J. E. Jones, N. A. G. Jones, R. M. Jones, Susan E. Jones, Keng, C. L. Kennedy, N. D. Kennedy, B. R. Kenning, A. S. T. Lamb, P. Laverick, R. D. Leach, Deborah L. J. Longstaff, S. M. Lord, A. G. Lyons, C. R. MacCallum, K. J. McGhee, J. K. Mackenzie, Anne Malleson, M. J. Maloney, R. Manna, P. G. Manning, B. J. Mansbridge, R. E. Mansel, M. A. Mansell,
C. A. S. Marrin, F. W. Martin, C. Mason, R. B. S. Mason, M. R. Masser, P. N. Matthews, P. C. Mattinglv,
R. E. G. Maxim, Annabel J. May, J. P. Maynard,
Renice Mendelson, C. S. H. Middleton, C. M. H.
Miller Jones, W'. J. Mintowt-Czyz, J. L. Mitchell, D. A. Monaghan Addy, R. Moore, R. C. Moore, G. M. Morgan, R. Morgan, Susan C. Morgan, Jacque-
line E. Morris, P. Morris, A. B. Morrison, A. L. H. Moss, R. W. Motson, J. M. Muir, D. C. Munday, Foh, K. W. Nightingale, G. C. K. Nkwazi, S. C. Norton, A. Z. S. Novak, Lesley J. Nuttall, Janine M. O'Kane, D. H. Oram, J. P. Osborne, J. R. Owen, A. J. F. Page, T. G. Palferman, Ann L. Parke, N. R. T. Pashley,
F. W. N. Paterson, C. A. Pearson, J. G. Pearson,
Nanette M. Pearson, M. H. Pelmore, R. A. Pennells, Nanette M. Pearson, M. H. Pelmore, R. A. Pennells,
G. Peters, J. M. Pharaoh, G. B. Piercy, A. L G. Peters, J. M. Pharaoh, G. B. Piercy, A. L. R. J. Powell, Mary J. Prentice, Catherine M. Price, J. E. Pring, C. K. W. Pugh, J. A. G. Punt, J. A. Quant, J. Rawlinson, R. A. Rayner, Janet K. Read, Janice W. Richardson, G. N. Richmond-Peck, Anne Rickerby, G. L. Ridgway, G. V. Rigby, N. Rizvi, C. D. Roberts, 1. Roberts, D. W. Robins, A. H. Robinson, C. Robinson, Linda J. Rockall, G. D. Rooker, Patricia A. Ross, P. G. Rudd, A. C. Ruddle, G. J. S. Rustin, J. E. Ryder, M. G. Sadler, P. T. R. Saunders, R. H. Savage, Kathrine M. P. L. Segal, D. R. J. Seingry, w. P. Seward, Margaret M. Shackell, T. H. Shepherd, Gillian M. P. Andrea G. Smith, C. C. Smith, Gillian E. Smith, M. H. Smith, M. J. Smith, J. O. Soul, D. P. Southall, Elizabeth J. Southcott, Elizabeth M. Spalding, R. S.
Stanley, P. J. Steer, Roslyn A. Stephens, A. J. Suggett,
Anne Sullivan, Valerie J. Sullivan, Anthea J. Swanton, Anne Sullivan, Valerie J. Sullivan, Anthea J. Swanton,
June R. Swinhoe, Pamela J. Taylor, Susan Taylor, Celia M. L. Teare, M. Thirlwall, D. J. B. Thomas, T. LI Thomas, Kay Thompson, R. G. N. Thonet, Rosalind J T. R. Tickner, V. L. R. Touquet, R. L. S. Toussaint, Ann J. Trent, J. K. Trivedi, I. F. Trotman, P. M. Trust, W. R. Tudor-Thomas, D. A. Turner, Jane M. Turner, P. J. Vickers, J. R. L. Vincent-Townend, D. A. Viniker, A. C. H. Vivian, Hilary Wade, P. J. F. Wade, S. J Marguerite C. Warburton, R. D. Ward, J. A. H Wass, D. G. Watson, Deirdre C. T. Watson, R. N Barbara Y. Wickson, G. R. Williams, W. A. Williamson, Wood, P. L. R. Wood, R. H. Wood, Jill P. J. Wright,

Zuckerman.
${ }^{1}$ With honours. guished in medicine. ${ }^{4}$ Distinguished in applied pharma"Distinguished in obstetrics and gynaecology.

LIVERPOOL

Appointments.-Dr. G. E. Foster (lecturer in anament) in paediatric cardiology, department of child ment) in
health).

ROYAL COLLEGE OF SURGEONS IN IRELAND F.R.C.S.I.-P. J. Robinson, G. C. Slack, R. Y. Tan, Aye Tun.

\section{CORRECTIONS}

Myeloma Workshop

In the contribution by Professor A. Videbaek on "Unusual Cases of Myelomatosis" (8 May, p. 326) the last sentence in the penultimate paragraph should have read "In a second patient there was a monoclonal gamma-A component, type kappa, in the serum starting at level of $2.4 \mathrm{~g} / 100 \mathrm{ml}$; this had a specificity of ASH (antistreptococcus hyalruonidase) with a titre of $5 \frac{1}{2}$ million units."

\section{Post-hypoglycaemic Encephalopathy}

In the letter by Dr. S. Krauss (5 June, p. 591) we regret that there was a printer's error in the third line of the final paragraph. The sentence should have read "-for example, using the flicker fusion technique and autonomic responses recorded on a polygraph-"

\section{Notice to Authors}

When original articles and letters for publication are not submitted exclusively to the British Medical fournal this must be stated.

Correspondence on editorial business should be addressed to the Editor, British Medical fournal, B.M.A. House, Tavistock Square, London WC1H 9JR. Telephone: 01-387 4499. Telegrams : Aitiology, London, W.C.1.

Authors wanting reprints of their articles should notify the Publishing Manager, B.M.A House, Tavistock Square, WC1H 9JR, on receipt of proofs. 